\title{
Fundamental photon noise limit to radial velocity measurements
}

\author{
F. Bouchy, F. Pepe, and D. Queloz
}

Observatoire de Genève, 51 ch. des Maillettes, 1290 Sauverny, Switzerland

Received 27 March 2001 / Accepted 9 May 2001

\begin{abstract}
In the past 5 years, improvements in radial velocity measurements have led to discovery of extra-solar planets and progress in asteroseismology programs. Doppler measurements with high precision is close to the limit given by photon noise. In this paper the methodology to compute the fundamental limit of radial velocity measurement given by photon noise is presented and illustrated with a representative sample of synthetic solar-type stellar spectra. Stellar rotational broadening, instrumental spectral range as well as spectral resolution influences are also considered. This study is applied to two dedicated spectrographs in order to help the optimization of radial velocity programs. Current methods of Doppler calculation are discussed and compared.
\end{abstract}

Key words. techniques: radial velocities - instrumentation: spectrographs - stars: oscillations stars: planetary systems

\section{Introduction}

The challenge of measuring small changes in stellar radial velocities (RV) is met in the search for extra-solar planets and the stellar seismology. Recently, much progress has been made in RV measurement techniques leading to the first detections of extra-solar planets (Mayor \& Queloz 1995; see review made by Perryman 2000) and the first evidence of oscillation modes in solar-type stars other than the Sun (Martic et al. 1999; Bedding et al. 2001).

Reaching a level of RV precision near $1 \mathrm{~ms}^{-1}$ over a large number of targets is a challenge and permits very ambitious programs. All extra-solar planets with a mass of 0.1 Jupiter mass orbiting solar-like stars at less than 1 AU can be detected after one year of observations. Moreover, detection of big telluric planets like 10 Earth mass planets on orbits shorter than $0.1 \mathrm{AU}$ is possible. In addition, but at a completely different time scale, RV precision at $1 \mathrm{~m} \mathrm{~s}^{-1}$ allows one to measure the asteroseismological parameters of solar-like stars in order to constrain theories of stellar structure and evolution. Acoustic mode oscillations are expected to have amplitudes in the range from 0.1 to $1 \mathrm{~m} \mathrm{~s}^{-1}$ and frequency in the range from 0.5 to $5 \mathrm{mHz}$. Several consecutive nights of observation may be needed to obtain a frequency resolution sufficient to resolve individual modes.

Today, the best precision reached in RV measurements is of a few $\mathrm{ms}^{-1}$. In most cases this precision is limited by the photon noise. Some studies on the impact of photon noise on RV measurements can be found in

Send offprint requests to: F. Bouchy, e-mail: Francois.Bouchy@obs.unige.ch
Brown (1990), Murdoch \& Hearnshaw (1991), and Mazeh \& Zucker (1994). In Sect. 2 we present the analysis of the fundamental noise limitation developed by Connes (1985) which can be used to estimate this fundamental uncertainty on the measurement of the velocity change $\delta V_{\mathrm{RMS}}$. In this analysis, spectral profile and photon count are taken into account. From this analysis the quality factor is defined and computed in Sect. 3 for various synthetic solar-type stellar spectra. The influence on the quality factor of the spectral type, the rotational broadening $v \sin i$, the spectral range and the spectral resolution of the instrument used are investigated. In Sect. 4 fundamental uncertainties $\delta V_{\mathrm{RMS}}$ of various solar-type stars are computed for the spectrograph CORALIE installed at the $1.20-\mathrm{m}$ Euler Swiss telescope, La Silla, Chile (Queloz et al. 2000) and the future spectrograph HARPS to be installed at the 3.60-m ESO telescope, at La Silla, Chile (Pepe et al. 2000). This study may be helpful for the optimization of scientific programs conducted on these two dedicated instruments. Finally, in Sect. 5 we discuss and compare current methods of precise RV measurement.

\section{The fundamental noise limitation in the Doppler shift measurement}

An optimum technique that uses the full spectral information available has been proposed by Connes (1985) for calculating radial velocity changes. In this procedure, a quality factor $Q$ is defined for each spectrum in order to compute the fundamental uncertainty on the radial velocity measurement due to noise. 
We consider $A_{0}$, a digitalized and calibrated spectrum obtained at epoch 0 , hereafter called the "reference" epoch or epoch of zero radial velocity. This spectrum is considered as being noise free. For a given pixel $i$, we know the intensity $A_{0}(i)$ (in photoelectrons) and the wavelength $\lambda(i)$. At another epoch, the spectrum is stretched by a Doppler shift and becomes $A$. At this point we do not consider any extra noise from detector (bias, dark, flat field), background light or cosmic ray pollution. Therefore these two spectra have the same intensity level.

The Doppler shift is given by:

$\frac{\delta V}{c}=\frac{\delta \lambda}{\lambda}$.

For a Doppler shift that is small compared to the linewidth, the observable intensity change at a given pixel can be expressed by:

$A(i)-A_{0}(i)=\frac{\partial A_{0}(i)}{\partial \lambda(i)} \delta \lambda(i)=\frac{\partial A_{0}(i)}{\partial \lambda(i)} \frac{\delta V(i)}{c} \lambda(i)$.

The Doppler shift is then given by:

$$
\frac{\delta V(i)}{c}=\frac{A(i)-A_{0}(i)}{\lambda(i)\left(\partial A_{0}(i) / \partial \lambda(i)\right)} \text {. }
$$

This equation shows that the change of the velocity is measured through a change of intensity in the recorded spectrum. To increase the sensitivity of the measurement and to decrease the noise, the whole available spectral range must be used. Hence, the contribution of all pixels is summed considering an optimum weight $W(i)$.

$$
\frac{\delta V}{c}=\frac{\sum \frac{\delta V(i)}{c} W(i)}{\sum W(i)}
$$

The optimum weight is proportional to the inverse square of the individual dispersion $\left(X_{\mathrm{RMS}}\right.$ designates later on the dispersion of the quantity $X)$. The optimum weight $W(i)$ is given by:

$$
W(i)=\frac{1}{\left(\frac{\delta V_{\mathrm{RMS}}(i)}{c}\right)^{2}} \text {. }
$$

The individual dispersion of the velocity change measured in pixel $i$ is given from Eq. (3) by:

$$
\frac{\delta V_{\mathrm{RMS}}(i)}{c}=\frac{\left[A(i)-A_{0}(i)\right]_{\mathrm{RMS}}}{\lambda(i)\left(\partial A_{0}(i) / \partial \lambda(i)\right)} \text {. }
$$

The spectrum $A_{0}$ is considered noise free. The noise of the spectrum $A$ comes from the quadratic sum of the photon noise $\sqrt{A}$ and the detector noise $\sigma_{\mathrm{D}}$; hence:

$$
[A(i)]_{\mathrm{RMS}}=\sqrt{A(i)+\sigma_{\mathrm{D}}^{2}} .
$$

Considering that $A$ and $A_{0}$ have the same intensity level and the Doppler shift is small, we can set $A=A_{0}$. The optimum weight is then given by:

$$
W(i)=\frac{\lambda^{2}(i)\left(\partial A_{0}(i) / \partial \lambda(i)\right)^{2}}{A_{0}(i)+\sigma_{\mathrm{D}}^{2}} .
$$

At this stage, for practical application, the weighted function may be modified to eliminate unwanted lines, such as telluric absorption lines.

The velocity change $\delta V$ measured from the full spectral range is then given by:

$$
\frac{\delta V}{c}=\frac{\sum\left(A(i)-A_{0}(i)\right)\left(\frac{W(i)}{A_{0}(i)+\sigma_{\mathrm{D}}^{2}}\right)^{1 / 2}}{\sum W(i)}
$$

One recalls that this relation is accurate only if the Doppler shift remains small compared to the line-width.

Now let $\delta V_{\text {RMS }}$ designate the uncertainty on the velocity change $\delta V$. According to Eq. (5), $\delta V_{\text {RMS }}$ is given by:

$\frac{\delta V_{\mathrm{RMS}}}{c}=\frac{1}{\sqrt{\sum W(i)}}=\frac{1}{Q \sqrt{\sum A_{0}(i)}}$,

where $Q=\frac{\sqrt{\sum W(i)}}{\sqrt{\sum A_{0}(i)}}$.

We see that if we neglect the detector noise contribution, this factor $Q$ is independent of the flux. It is a sole function of the spectral profile within the spectral range considered. It represents the quality and the spectral line richness of the spectrum.

Considering that $\sum A_{0}(i)=N_{\mathrm{e}^{-}}$is the total number of photoelectrons counted over the whole spectral range, the uncertainty of the velocity change is finally given by:

$\delta V_{\mathrm{RMS}}=\frac{c}{Q \sqrt{N_{\mathrm{e}^{-}}}}$.

With the quality factor $Q$ and the total number of photoelectrons $N_{\mathrm{e}^{-}}$, the fundamental noise limitation in velocity measurements can be calculated. In cases of computation of this quantity for each spectral slice $k$ (or spectral order $k$ ) of the spectrum, a weighted average of all these slices must be computed as:

$\overline{\delta V_{\mathrm{RMS}}}=\frac{1}{\sqrt{\sum\left(\frac{1}{\delta V_{\mathrm{RMS}}(k)}\right)^{2}}}$.

This procedure was tested and demonstrated by Connes et al. (1996) using observations collected with the ELODIE spectrograph based on the 1.93-m telescope at the Observatoire de Haute Provence. It is also currently used on the EMILIE spectrograph (Bouchy et al. 1999) coupled to the Absolute Astronomical Accelerometer (Connes 1994; Schmitt 1997) installed on the 1.52-m telescope of the same observatory.

\section{Numerical quality factor of synthetic solar-type stellar spectra}

\subsection{On the effect of the spectral type}

The quality factor derived from Eq. (11) may be computed for any stellar spectrum. We have used synthetic spectra realized with the SPECTRUM code developed by 
Table 1. Parameters of synthetic spectra.

\begin{tabular}{|c|c|c|c|c|}
\hline $\begin{array}{c}\text { Spectral } \\
\text { Type }\end{array}$ & $\begin{array}{c}T_{\text {eff }} \\
(\mathrm{K})\end{array}$ & $\begin{array}{c}\log (g) \\
\left(\mathrm{cm} \mathrm{s}^{-2}\right)\end{array}$ & $\begin{array}{c}V_{\text {turb }} \\
\left(\mathrm{km} \mathrm{s}^{-1}\right)\end{array}$ & $\begin{array}{c}\text { Quality } \\
\text { Factor }\end{array}$ \\
\hline K7V & 4000 & 4.5 & 1.0 & 31020 \\
K5V & 4500 & 4.5 & 1.0 & 34940 \\
K2V & 5000 & 4.5 & 1.0 & 33405 \\
G8V & 5500 & 4.5 & 1.0 & 30375 \\
F9V & 6000 & 4.5 & 1.25 & 24450 \\
F5V & 6500 & 4.5 & 1.5 & 19250 \\
F2V & 7000 & 4.5 & 2.0 & 14430 \\
\hline
\end{tabular}

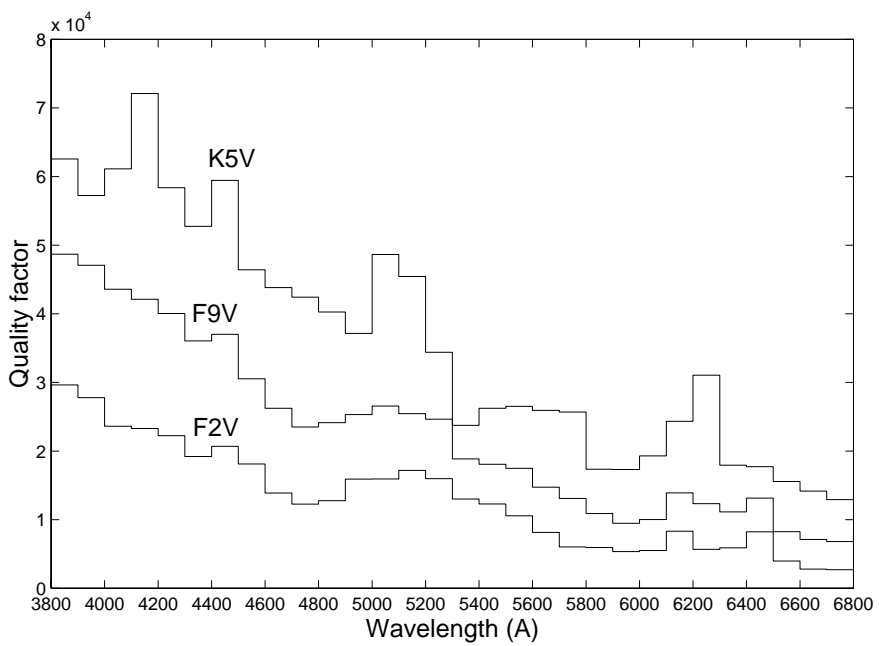

Fig. 1. Quality factor versus spectral range for a $\mathrm{K} 5 \mathrm{~V}, \mathrm{~F} 9 \mathrm{~V}$ and F2V star.

R. O. Gray (Gray \& Corbally 1994) using the stellar atmosphere models of Kurucz (1994). The study was restricted to dwarf stars with spectral type in the range $\mathrm{F} 2 \mathrm{~V}$ to $\mathrm{K} 7 \mathrm{~V}$ with solar abundances. These stars are ideal targets for a precise RV measurement campaign. Earlier type stars have few lines and those lines are usually broadened by high rotational velocity.

Table 1 presents the parameters of the 7 synthetic spectra with their quality factors computed in the spectral range from 3800 to $6800 \AA$ at an infinite spectral resolution (sampling $5 \mathrm{~m} \AA$ ). The best quality factor appears to be for a K5V star and decreases by more than a factor of 2 for a $\mathrm{F} 2 \mathrm{~V}$ star.

\subsection{On the effect of the wavelength spectral range}

To examine the influence of the spectral range, the quality factors was computed separately for a set of small spectral slices of $100 \AA$. The results for a K5V, F9V and F2V star are presented in Fig. 1. The quality factor decreases by about a factor 6 between the blue and the red part of the spectra.

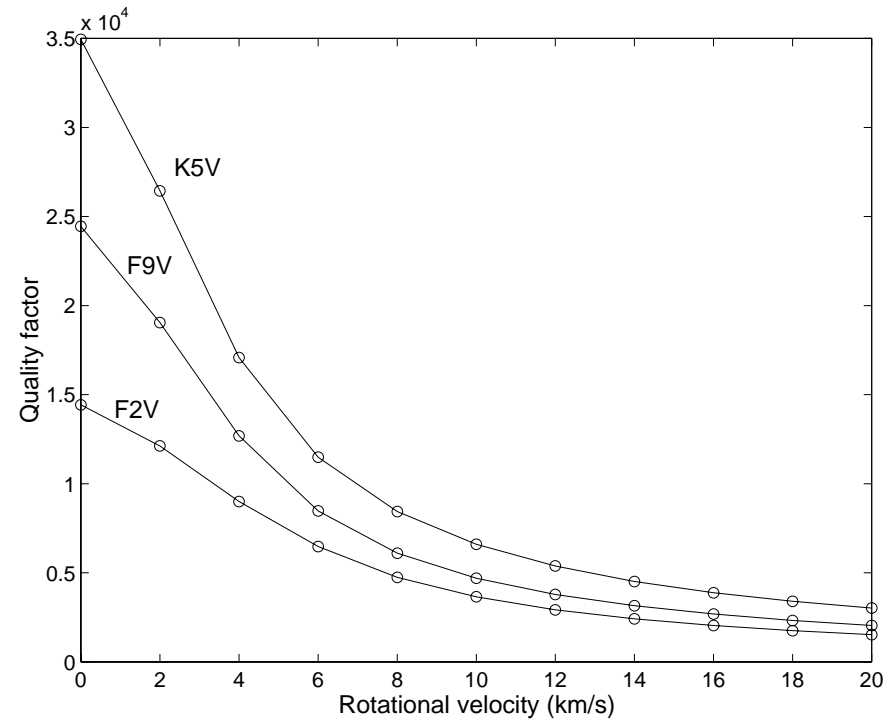

Fig. 2. Quality factor versus rotational broadening $v \sin i$ for a $\mathrm{K} 5 \mathrm{~V}, \mathrm{~F} 9 \mathrm{~V}$, and F2V star.

\subsection{On the effect of the rotational broadening}

Line rotational velocity broadening degrades the quality factor. Fast rotators are often associated with early type star. Note that these stars are often detected as intrinsically variable (Saar \& Donahue 1997).

Table 2 presents the numerical quality factor for the 7 synthetic spectra computed over the whole spectral range for different $v \sin i$. Two typical behaviors can be distinguished. For $v \sin i$ values less than $6 \mathrm{kms}^{-1}$, lines profile is only broadened by rotational velocity and $Q$ decreases linearly with $v \sin i$. For $v \sin i$ greater than $6 \mathrm{~km} \mathrm{~s}^{-1}$, line profiles begin to be significantly modified by the blending effect of neighboring spectral lines. In this case, $Q$ is proportional to $v \sin i^{-1}$. Figure 2 illustrates the quality factor versus $v \sin i$ for a K5V, F9V and F2V star.

\subsection{On the influence of the spectrograph resolution}

The spectrograph resolution limits the stellar spectrum quality factor as long as the stellar lines are not resolved. Figure 3 shows the quality factor computed for the whole spectral range versus spectral resolution (spectra are convolved with a Gaussian profile with $F W H M=\lambda / R$ ) for a K5V, F9V and F2V star in two cases of projected rotational velocity: $v \sin i=0$ and $v \sin i=10 \mathrm{~km} \mathrm{~s}^{-1}$. This figure shows that there is advantage to resolving lines beyond 50000 but a resolution greater than 100000 is interesting only in a few cases of stellar spectra. Similar to the $v \sin i$ dependency described above, two distinct rates appear. For $R$ lower than 50000 , all the lines are blended and $Q$ increases linearly with $R$. For $R$ greater than 50000 , lines are more and more resolved and the relation flatness becomes linearly decreasing with $R^{-1}$. 
Table 2. Quality factor versus rotational broadening $v \sin i$ for the 7 synthetic spectra.

\begin{tabular}{|c|c|c|c|c|c|c|}
\hline Spectral Type & $0 \mathrm{~km} \mathrm{~s}^{-1}$ & $4 \mathrm{~km} \mathrm{~s}^{-1}$ & $\begin{array}{c}v \sin i \\
8 \mathrm{~km} \mathrm{~s}^{-1}\end{array}$ & $12 \mathrm{~km} \mathrm{~s}^{-1}$ & $16 \mathrm{~km} \mathrm{~s}^{-1}$ & $20 \mathrm{~km} \mathrm{~s}^{-1}$ \\
\hline K7V & 31150 & 15605 & 8015 & 5185 & 3785 & 2975 \\
K5V & 34940 & 17080 & 8440 & 5380 & 3885 & 3020 \\
K2V & 33445 & 16140 & 7815 & 4930 & 3545 & 2740 \\
G8V & 30415 & 14700 & 7020 & 4385 & 3140 & 2410 \\
F9V & 24450 & 12685 & 6105 & 3785 & 2690 & 2045 \\
F5V & 19245 & 10670 & 5240 & 3230 & 2270 & 1715 \\
F2V & 14430 & 9000 & 4750 & 2925 & 2045 & 1530 \\
\hline
\end{tabular}

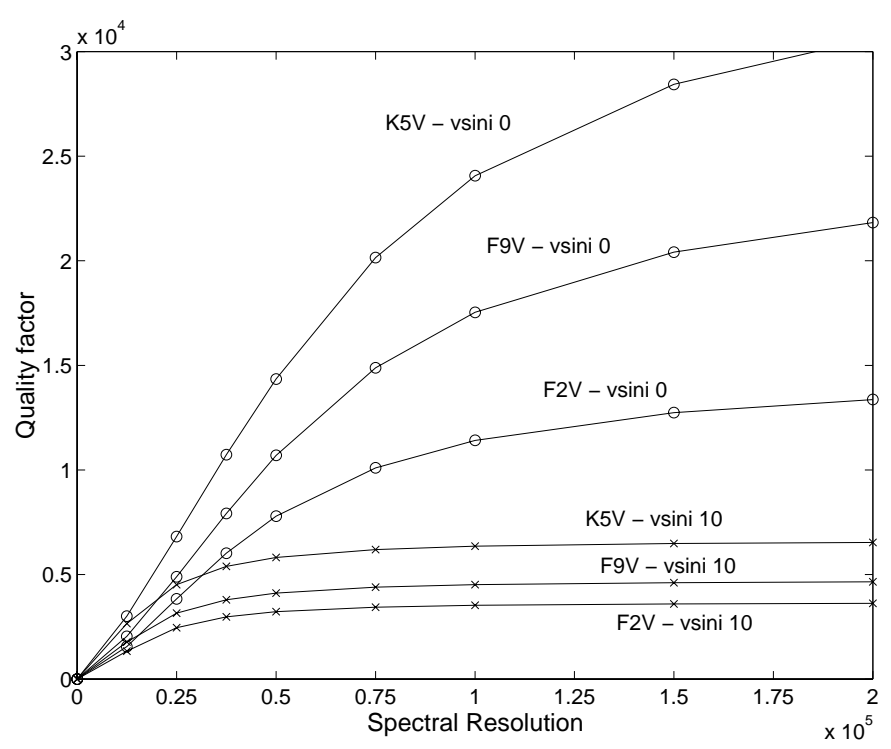

Fig. 3. Quality factor versus spectrograph resolution for a $\mathrm{K} 5 \mathrm{~V}, \mathrm{~F} 9 \mathrm{~V}$, and $\mathrm{F} 2 \mathrm{~V}$ star with $v \sin i=0$ and $v \sin i=$ $10 \mathrm{~km} \mathrm{~s}^{-1}$.

\section{The radial velocity uncertainty for some spectrographs}

\subsection{The method}

To compute the fundamental uncertainty on RV by Eq. (12), we need to estimate the total number of photoelectrons collected over the spectral range used. Allen (1973) gives the spectral flux of $m v=0$ stars outside the Earth's atmosphere. Figure 4 show this spectral flux interpolated and integrated for $100 \AA$ slices of the spectral range from 3800 to $6800 \AA$ for a F0V, G0V, K0V and M0V star. We interpolate from these curves the spectral flux for the 7 synthetic spectra of Table 1 . This quantity $F_{\star}$ is expressed in photon $\mathrm{cm}^{-2} \mathrm{~s}^{-1}$. The total number of photoelectrons counted over each slice of $100 \AA$ is then given by:

$N_{\mathrm{e}^{-}}=\frac{F_{\star} \cdot S_{\mathrm{tel}} \cdot e_{\mathrm{tot}} \cdot t_{\mathrm{exp}}}{2.512^{m v}}$,

with $S_{\text {tel }}$ the telescope area in $\mathrm{cm}^{2}$, $e_{\text {tot }}$ the total efficiency including atmosphere, telescope, spectrograph and detector, $t_{\exp }$ the exposure time in $\mathrm{s}$, and $m v$ the visual magnitude of the star.

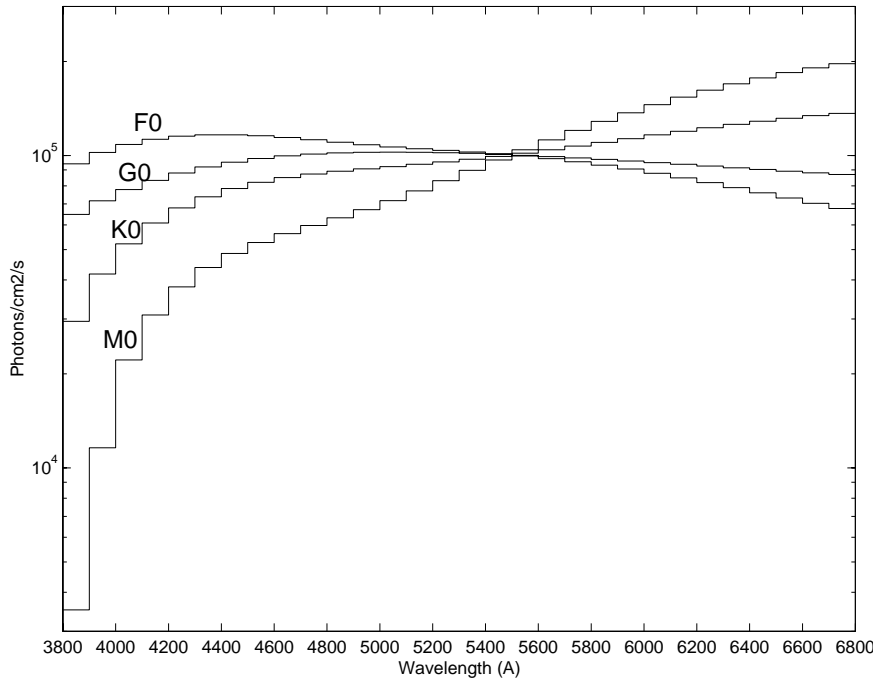

Fig. 4. Stellar flux outside the Earth's atmosphere integrated on a $100 \AA$ slice for a F0V, G0V, K0V and M0V star of $m v=0$.

The uncertainty of the RV change is then given by:

$\delta V_{\mathrm{RMS}}=\delta V_{\mathrm{RMS} 0} \cdot \sqrt{\frac{2.512^{m v}}{t_{\exp }}}$,

with $\delta V_{\mathrm{RMSO}}=\frac{c}{Q \sqrt{F_{\star} \cdot S_{\mathrm{tel}} \cdot e_{\mathrm{tot}}}}$.

The quantity $\delta V_{\text {RMSo }}$ represents the fundamental radial velocity uncertainty due to the photon noise for $t_{\exp }(s)=$ $2.512^{m v}$.

Quality factors $Q$ are computed as described in Sect. 3 for all the 7 synthetic spectra with different rotational velocities, taking into account the spectral resolution and total efficiency of the instrument. We have neglected in this study the detector noise (which is justified for high $S / N$ ratios) and we have attempted to use the whole spectral range from 3800 to $6800 \AA$.

\subsection{The CORALIE spectrograph}

CORALIE (Queloz et al. 2000) is the southern hemisphere twin of the ELODIE spectrograph (Baranne et al. 1996), mounted on the 1.2-m Euler Swiss telescope at La Silla, 


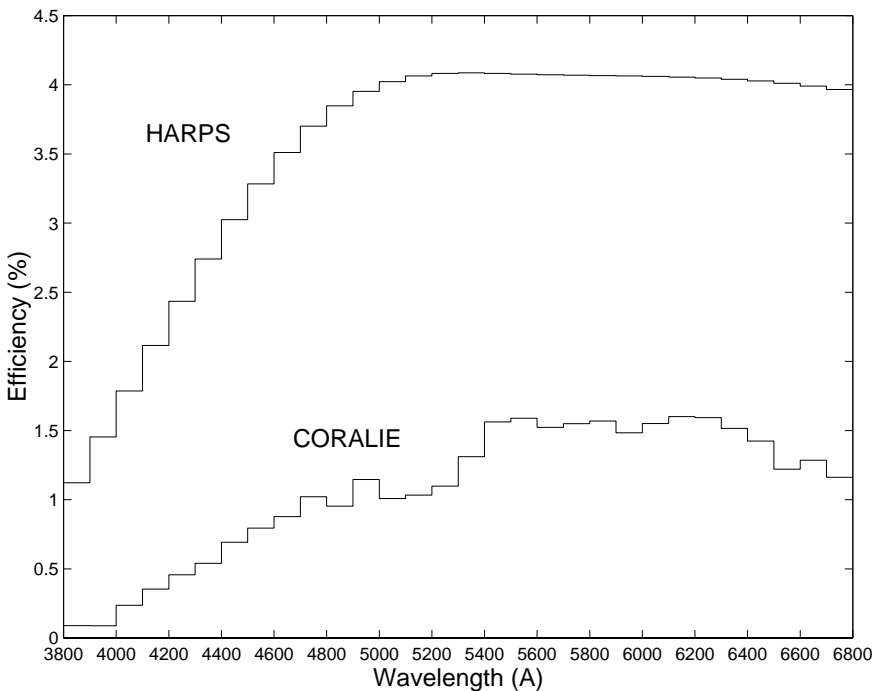

Fig. 5. Total efficiency measured with CORALIE in a medium seeing condition and the same value estimated for HARPS.

Chile. Wavelength covered ranges from 3875 to $6820 \AA$ with a spectral resolution of 50000 . The total efficiency over the whole spectral range including atmosphere, telescope, spectrograph and detector is presented in Fig. 5. The quantity $\delta V_{\text {RMSo }}$ is presented in Fig. 6 . In the best stellar case $\left(T_{\text {eff }}=4500 \mathrm{~K}, v \sin i=0 \mathrm{~km} \mathrm{~s}^{-1}\right)$, the RV uncertainty of $1 \mathrm{~ms}^{-1}$ is obtained on a 6 magnitude star with 8 minutes exposure time.

The RV uncertainty of a real spectrum obtained with CORALIE is compared with the expected one deduced from synthetic spectra. We use a spectrum of the star HD 83443 where two extra-solar planets have been detected (Mayor et al. 2000). HD 83443 is a K0V star with a visible magnitude $m v=8.23$ and projected rotational velocity $v \sin i=1.9 \mathrm{~km} \mathrm{~s}^{-1}$. With a $750 \mathrm{~s}$ exposure, we expect from Fig. 6 a fundamental uncertainty of about $2.6 \mathrm{~m} \mathrm{~s}^{-1}$. To compute the RV uncertainty of the observed spectrum, we clean it from cosmic ray hits and we limit its spectral range from 3994 to $6820 \AA$ to avoid orders where detector noise is greater than photon noise. The result is $2.93 \mathrm{~m} \mathrm{~s}^{-1}$ and compares well with the expected value. In addition, a Monte Carlo noise simulation (including photon and detector noise) is made on this observed spectrum. The optimum weight procedure described in Sect. 2 is used to compute the radial velocity. The radial velocity dispersion reaches $2.96 \mathrm{~m} \mathrm{~s}^{-1}$, in full agreement with the calculated uncertainty.

A sequence of RV measurements on the Sun is presented to demonstrate that the estimated errors on the RV measurements are in agreement with the observations. During the measurements a diffusing mask was inserted at the entrance of the telescope to obtain a full integrated solar disk and to be free of any possible guiding errors. On the RV measurement sequence presented in Fig. 7, we measure a dispersion of $0.55 \mathrm{~m} \mathrm{~s}^{-1}$. The estimated errors due to photon noise on the RV measurements stemming from the simultaneous thorium spectrum used in the in-

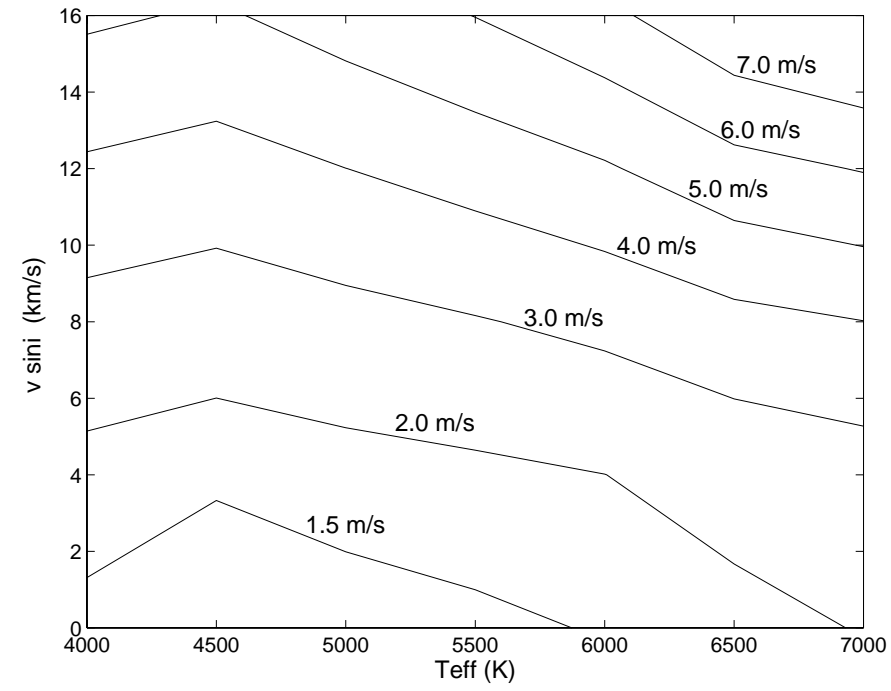

Fig. 6. Radial velocity uncertainty predictions for CORALIE $\left(t_{\exp }(s)=2.512^{m v}\right)$.

strumental tracking is about $0.30 \mathrm{~m} \mathrm{~s}^{-1}$. The photon noise error stemming from the stellar flux reaches $0.38 \mathrm{~m} \mathrm{~s}^{-1}$. If we take into account an extra signal of about $0.25 \mathrm{~m} \mathrm{~s}^{-1}$ coming from the 5-mn oscillation modes of the Sun, we are in agreement with the $0.55 \mathrm{~m} \mathrm{~s}^{-1}$ observed value. This "real life test" demonstrated that precision better that $1 \mathrm{~m} \mathrm{~s}^{-1}$ is achievable on CORALIE and that the proposed algorithm provides a very efficient way to extract the RV information from a stellar spectrum.

\subsection{The HARPS spectrograph}

The HARPS spectrograph (Pepe et al. 2000) will be installed on the ESO 3.6-m telescope at La Silla, Chile. It will start its operations at the end of 2002. The wavelength spectral domain ranges from 3770 to $6900 \AA$ with a spectral resolution of 90000 . Its estimated total throughput including atmosphere, telescope, spectrograph and detector is presented in Fig. 5. Its estimated $\delta V_{\mathrm{RMSO}}$ is presented in Fig. 8.

Compared to CORALIE, the gain on RV uncertainty reaches a factor of 7 to 9 . This means that for a given RV fundamental limit, HARPS will need exposure times 49 to 81 times shorter or it will observe stars with magnitudes 4.2 to 4.8 dimmer. Figure 9 shows the RV uncertainty $\delta V_{\text {RMS }}$ versus stellar magnitude computed for HARPS for the best stellar spectrum case $\left(T_{\text {eff }}=4500 \mathrm{~K}, v \sin i=\right.$ $0 \mathrm{~km} \mathrm{~s}^{-1}$ ) for various exposure times. Considering the conversion factor of the CCD $\left(1.64 \mathrm{e}^{-} / \mathrm{ADU}\right)$, the saturation level of the CCD converter is reached for a spectral $S / N$ corresponding to a $\delta V_{\mathrm{RMS}}=0.05 \mathrm{~m} \mathrm{~s}^{-1}$. In the case of low $S / N$ spectrum $(S / N<10)$, detector read-out noise $\left(4.7 \mathrm{e}^{-}\right)$should be taken into account. This $S / N$ domain corresponds to $\delta V_{\mathrm{RMS}}>3 \mathrm{~m} \mathrm{~s}^{-1}$. These respectively upper and lower radial velocity limits define the range of parameters illustrated in Fig. 9. 


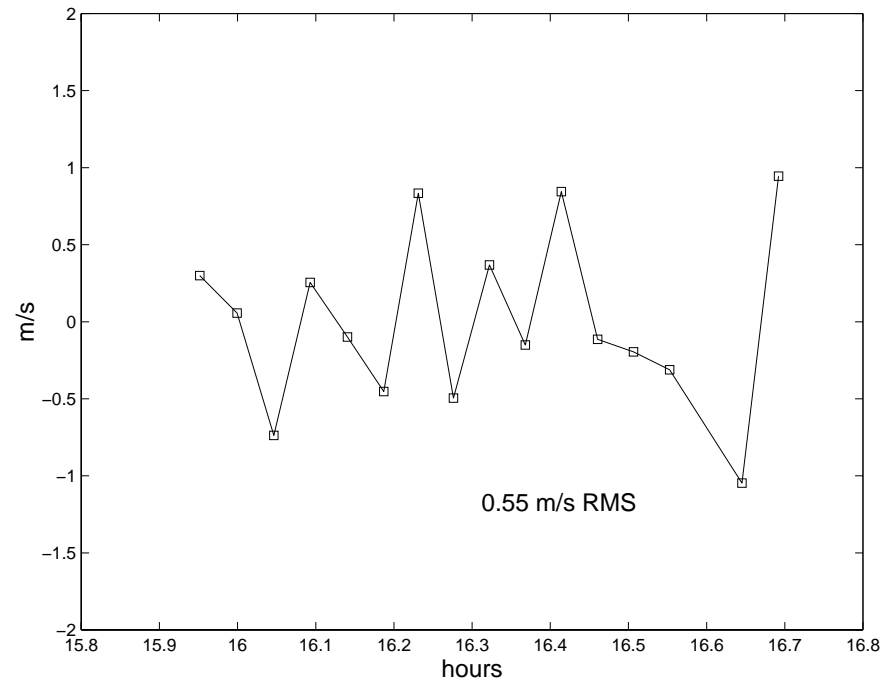

Fig. 7. Sequence of VR measurements carried out with CORALIE on the integrated solar disk using the algorithm presented in this paper. The sequence lasts $45 \mathrm{mn}$ and is made of 1-mn exposures. The observed $0.55 \mathrm{~m} \mathrm{~s}^{-1} \mathrm{rms}$ agrees well with the expected dispersion stemming from photon noise.

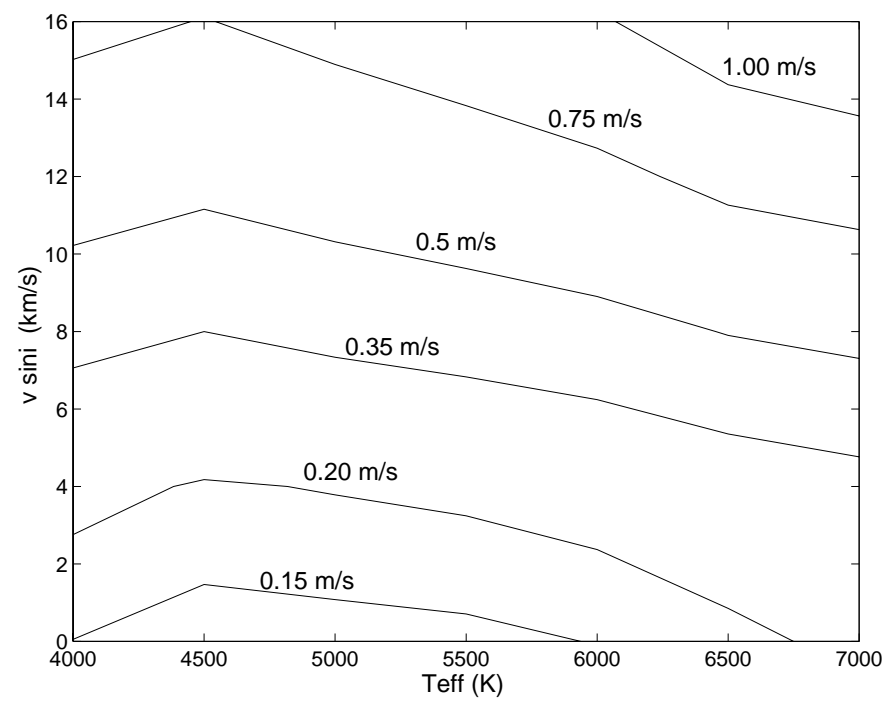

Fig. 8. Radial velocity uncertainty predictions for HARPS $\left(t_{\exp }(s)=2.512^{m v}\right)$.

\section{Discussions}

Essentially two different methods are used to gather precise RV measurements. Both track instrumental changes by comparison with a set of stable reference lines. One method, called the double-fiber or simultaneous wavelength reference method, makes use of an optical fiber for the starlight, and a second fiber illuminated by a stable laboratory source (usually a thorium lamp) (see Baranne et al. 1996 for details). The other method, called the Iodine cell method, inserts in the input stellar beam an iodine cell to superimpose stable absorption lines on the incoming starlight (see Butler \& Marcy 1996 for details). The observation is modeled as the product of two functions, the intrinsic stellar spectrum and the transmission function of

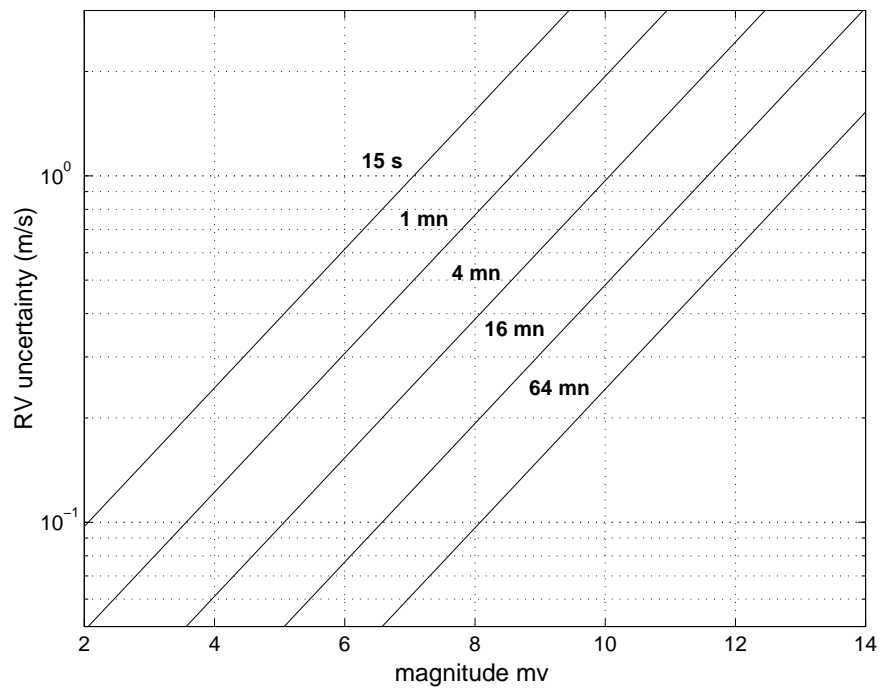

Fig. 9. Radial velocity uncertainty predictions for HARPS versus magnitude for a star spectrum with $T_{\text {eff }}=4500 \mathrm{~K}$ and $v \sin i=0 \mathrm{kms}^{-1}$.

the iodine absorption cell. The model requires 13 parameters, 2 for the wavelength scale, 1 for the Doppler shift, and 10 for the spectrograph PSF description. With this technique the spectral range is limited between 5000 and $6300 \AA$ and the total efficiency is reduced by a factor of $\sim 2$ by the effect of the $I_{2}$ line forest. We compute the fundamental RV uncertainty on the stellar spectrum taking into account the average transmission of the UVES iodine cell (Kurster 1999) in the limited spectral range. In this computation, the deconvolution process is considered as noise-free. We find that fundamental photon uncertainties on radial velocity measurements done by the iodine cell technique is greater by a factor 2 to 2.4 compared to the double-fiber method. This means that for a given RV fundamental limit, a program using the iodine cell mode needs exposure time 4 to 6 times greater or a telescope with an aperture diameter at least 2 times larger.

The present procedure used on CORALIE to estimate the variation of RV is based on a cross-correlation technique that numerically replicates the optical process carried out by the mask of CORAVEL (see Baranne et al. 1979 for details). The wavelength shift is measured by fitting a Gaussian function to the cross correlation of the stellar spectrum with a binary mask made of box-shaped emission lines of equal amplitude. To compare the efficiency of the optimum weight procedure with the crosscorrelation procedure, we have run a Monte Carlo noise simulation similar to the one described in Sect. 4.2. We find that the optimum procedure is a factor 1.6 more efficient. Actually, the cross-correlation method is far from optimum. The box-shaped emission lines of the binary mask are not associated with all the real spectral lines (i.e. the full spectral information is not used). Moreover, no optimum weighting task is carried out. This effect was already discussed by Chelli (2000), who compared an 
optimized algorithm based on a data analysis in the spectral Fourier space with the cross-correlation method.

\section{Concluding remarks}

This study has shown that photon noise permits measurement of stellar radial velocities at a precision level of $1 \mathrm{~m} \mathrm{~s}^{-1}$. This precision is necessary to conduct ambitious programs in the search for extra-solar planets and for asteroseismology. In this paper, the complete methodology to compute in every case the fundamental photon noise limit in RV measurements was presented. The optimum weight procedure for calculating RV changes was developed and compared to other methods currently used.

We are aware that the limits on RV measurements are also set by instrumental instabilities. However, recent tests carried on with the EMILIE and CORALIE spectrographs on laboratory sources and the Sun show an intrinsic short term stability at a level lower than $1 \mathrm{~m} \mathrm{~s}^{-1}$. At this time, the long term precision reached with CORALIE is close to $2 \mathrm{~m} \mathrm{~s}^{-1}$ (Queloz et al. in preparation). Strong efforts are made to increase the intrinsic stability of HARPS in order to reach the $1 \mathrm{~m} \mathrm{~s}^{-1}$ precision level.

Extra-solar planetary search programs can be limited by the intrinsic stellar RV variability induced by motion of the photosphere due to pulsation and/or stellar activityrelated variations, like rotation of star spots or convective inhomogeneities and their temporal evolution. Knowledge of the intrinsic stability of stars at the level of $1 \mathrm{~m} \mathrm{~s}^{-1}$ is still limited but a clear relation between stellar RV variability and rotational broadening $v \sin i$ has been shown (Saar et al. 1998; Santos et al. 2000). An empirical limit at $v \sin i=4 \mathrm{~km} \mathrm{~s}^{-1}$ has been fixed for most extra-solar planetary search programs. Note that this limitation does not appear in asteroseismology programs since we try to characterize intrinsic activity of stars over the time scale from a few minutes to one hour.

Acknowledgements. We are grateful to D. Espamer who helped us in the use of the SPECTRUM code. We wish to thank the Geneva University, the Swiss National Science Foundation (FNRS) and the Federal Office for Science and Education for the continuous support for CORALIE and HARPS projects.

\section{References}

Allen, C. W. 1973, Astrophysical Quantities 3rd ed., Univ. of London (Athlone Press)

Baranne, A., Mayor, M., \& Poncet, J. L. 1979, Vistas Astron., 23,279

Baranne, A., Queloz, D., Mayor, M., et al. 1996, A\&AS, 119, 373

Bedding, T. R., Butler, R. P., Kjeldsen, H., et al. 2001, ApJ, 549, L105

Bouchy, F., Connes, P., Bertaux, J.-L. 1999, in Precise Stellar Radial Velocities, IAU Coll. 170, ed. J. B. Hearnshaw, \& C. D. Scarfe, ASP Conf. Ser., 185, 22

Brown, T. M. 1990, in CCDs in Astronomy, ed. G. Jacoby, ASP Conf. Ser., 8, 335

Butler, R. P., Marcy, G. W., Williams, E., et al. 1996, PASP, 108,500

Chelli, A. 2000, A\&A, 358, L59

Connes, P. 1985, Astrophys. Space Sci., 110, 211

Connes, P. 1994, Astrophys. Space Sci., 212, 357

Connes, P., Martic, M., \& Schmitt, J. 1996, Astrophys. Space Sci., 241, 61

Gray, R. O., \& Corbally, C. J. 1994, AJ, 107, 742

Kurster, M. 1999, Properties of the UVES iodine cells, Internal ESO Memorandum

Kurucz, R. L. 1994, CD-ROM-19, Smothsoniam Astrophys. Obs. (Cambridge, MA)

Martic, M., Schmitt, J., Lebrun, J.-C., et al. 1999, A\&A, 351, 993

Mayor, M., \& Queloz, D. 1995, Nature, 378, 355

Mayor, M., Naef, D., Pepe, F., et al. 2000, in Planetary Systems in the Universe: Observation, Formation and Evolution, IAU Symp. 202, ed. A. J. Penny, P. Artymowicz, A.-M. Lagrange, \& S. S. Russel, ASP Conf. Ser., in press

Mazeh, T., \& Zucker, S. 1994, Astrophys. Space Sci., 212, 349

Murdoch, K., \& Hearnshaw, J. B. 1991, Astrophys. Space Sci., 186,137

Pepe, F., Mayor, M., Delabre, B., et al. 2000, Proc. SPIE, 4008, 582

Perryman, M. A. C. 2000, Rep. Prog. Phys., 63, 1209

Queloz, D., Mayor, M., Weber, L., et al. 2000, A\&A, 354, 99

Saar, S. H., \& Donahue, R. A. 1997, ApJ, 485, 319

Saar, S. H., Butler, R. P., \& Marcy, G. W. 1998, ApJ, 498, $\mathrm{L} 153$

Santos, N. C., Mayor, M., Naef, D., et al. 2000, A\&A, 361, 265

Schmitt, J. 1997, Thesis University Paris 6 